\title{
Trauma und Gewalt
}

Einleitung in den Schwerpunkt

Dass Trauma - das griechische Wort für Wunde - gerade nicht nur eine individuelle seelische Störung von Menschen darstellt, sondern auch als Symptom einer gesellschaftlichen Störung zu begreifen ist, hat die gesellschaftliche Aufregung bei Bekanntwerden der unsäglich leidvollen (sexuellen) Gewalterfahrungen von Kindern und Jugendlichen in kirchlichen und (reform-) pädagogischen Einrichtungen wieder mehr ins Bewusstsein gebracht. Erkennbar wurde nämlich, dass Traumatisierung ein Einwirken sozialer Kräfte auf das Individuum bezeichnet, das hätte verhindert werden können und sollen.

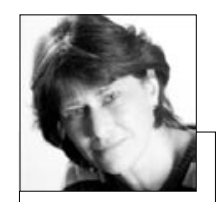

Margret Dörr *1956

Dr., Professorin an der schule für Soziale Arbeit in Mainz. Arbeitsschwerpunkte: Theorien Sozialer Arbeit; Biographieund Sozialisationstheorie, Psychoanalytische (Sozial-) Pädagogik. margret.doerr @t-online.de Katholischen Fachhoch-

Trauma bzw. Traumatisierung von Menschen ist daher keineswegs ein nur klinisch relevanter Begriff, sondern ebenso ein soziales und politisches Phänomen. Als Regel kann dabei gelten, dass Individuen traumatischen Ereignissen deshalb ausgesetzt waren (und immer noch sind), weil sie Angehörige von besonders verwundbaren sozialen Gruppen sind (Kinder, insb. Heimkinder, Asylbewerber, Flüchtlinge, Frauen, arme Menschen). Insofern ist auch die Thematisierung der vielfältigen Leiden von (sexueller) Gewalt betroffener Kinder, Jugendliche und erwachsene Menschen ohne die radikale Einbeziehung der Verfasstheit unserer gesellschaftlichen Institutionen nicht angemessen. Denn, wie MARGALIT (1999) pointiert: Eine gute Gesellschaft ist eine anständige Gesellschaft, und eine Gesellschaft ist anständig, wenn ihre Institutionen die Menschen nicht demütigen.

Wie die Institution Soziale Arbeit - als in Strukturen geronnenes Denk-, Handlungs- und Beziehungsmuster - auf die gesellschaftlich relevante Frage in einer Demokratie: „Was tun mit traumatisierten Menschen?" antwortet, um sich in ihrer professionellen Praxis der gesellschaftli- chen Verantwortung zu stellen, ohne die Betroffenen allein einer klinisch-psychologischen Profession zu überantworten, wird am Beispiel ausgewählter Aspekte erörtert. So zeigt Martin Kühn wesentliche Dimensionen der „Traumapädagogik“ in stationären Jugendhilfeeinrichtungen auf und vermag mit seiner Konzeption einer „Pädagogik des Sicheren Ortes“ anschaulich zu machen, wie sehr die besondere Verantwortung der Jugendhilfe in der Konstitution und Aufrechterhaltung von sicheren Lebensorten liegt. Jene „Sicheren Orte“ sind nicht nur Voraussetzung dafür, dass traumatisierte - verstörte, haltlose und oftmals störende - Kinder und Jugendliche hinreichende Chancen zur (Wieder-) Gewinnung von Selbstwirksamkeitserfahrungen erhalten. Sie stellen ebenso eine zentrale Basis für PädagogInnen dar, um den hohen Anforderungen eines haltgebenden „Geschützten Dialogs“"mit selbst- und weltverzweifelten Kindern und Jugendlichen gerecht werden zu können. Ulrike Loch und Heidrun Schulze wenden sich dezidiert gegen die noch immer weit verbreitete Einstellung, traumatisierte Menschen wären in erster Linie „kranke“ bzw. „gestörte“ Menschen und folglich allein AdressatInnen medizinischer und psychologischer Professionen.
Demgegenüber eröffnen die beiden Autorinnen in ihrem Beitrag einen Blick auf mögliche traumasensible (Handlungs-) Räume Sozialer Arbeit. Mit ihrem Plädoyer für eine „psychosoziale Traumatologie“ verweisen sie auf den hohen Rang der Sozialen Arbeit für ein gesellschaftlich verantwortliches Handeln mit traumatisierten Menschen, die noch zu wenig zum Selbstverständnis psychosozialer und pädagogischer Praxis im deutschsprachigen Raum gehört. Michaela Huber wendet sich der Frage nach der existenziellen Bedeutung von frühen Gewalterfahrungen bei vor allem jungen Kindern zu, erläutert die fundamentalen nachhaltigen Schädigungen für ihre weitere Entwicklung und macht eindringlich die Aufgabe einer Sozialen Arbeit deutlich, den betroffenen Familien mehr und frühere Hilfsangebote zu machen. Hierbei mahnt sie an, in jenen Fällen, in denen die Eltern ihr gewalttätiges Handeln gegen ihre Kinder trotz angemessener Unterstützung nicht aktiv ändern wollen, ihnen gegenüber konsequent einen Umgangsentzug durchzusetzen. Marianne Rauwald erläutert entlang einer kurzen Fallvignette, die als typisch für die (sozial-) pädagogische Arbeit mit (sexueller) Gewalt betroffener Kinder gelten kann, die komplizierten psychischen Mechanismen, die zum Phänomen der transgenerationalen Weitergabe von traumatischen Erfahrungen und darüber zu Entwicklungsstörungen bei ihren Kindern führen. So kann sie erhellen, wie Eltern ihre selbst erlebten (sexuellen) Gewalterfahrungen - gegen ihre bewusste Absicht - an ihre Kinder weitergeben. Damit Eltern ihrem Wunsch, für sich und ihre Kinder eine von der eigenen Gewaltgeschichte befreite Zukunft zu gestalten, umsetzen können, benötigen diese die Chance, sich mit ihren chronifizierten Folgen eigener Traumatisierungen auseinander zu setzen.
Literatur

MARGALIT, A. (1999).

Politik der Würde. Über Achtung und Verachtung. Frankfurt/M. 